\title{
A highlight of recent advances in immunology
}

\author{
ZHENG DeXian \\ National Laboratory of Medical Molecular Medicine, Institute of Basic Medical Sciences, Chinese Academy of Medical Sciences, \\ Beijing 100005, China
}

Received October 20, 2011; accepted November 10, 2011

Citation: Zheng D X. A highlight of recent advances in immunology. Sci China Life Sci, 2011, 54: 1151-1152, doi: 10.1007/s11427-011-4251-1

To celebrate the 60th anniversary of SCIENCE CHINA, six research groups of overseas and domestic Chinese immunologists published a series of review articles (SCIENCE CHINA Life Sciences, 2010, 53(2): 157-158), which highlighted recent advances and their contributions to immunology.

Wang YaYa in Prof. Cheng GenHong's group, who discovered the function of TNF receptor-associated factor (TRAF) and other signal molecules in Toll-like receptor (TLR) mediated signaling pathway and innate immunity [1], reviewed TRAF-mediated regulation of immune and inflammatory responses [2]. TRAF family consists of six mammalian members (TRAF1, TRAF2, TRAF3, TRAF4, TRAF5, and TRAF6) and participates in signal transduction of a large number of receptor families such as TNF receptor family (TNFR) and TLR-interleukin-1 receptor (TLR-IL-1R) family. Upon receptor-mediated activation, TRAFs are directly or indirectly recruited to the intracellular domains of these receptors and subsequently combine with other signaling molecules to activate the inhibitor of I $\mathrm{B}$ kinase (IKK) complex, TRAF family member-associated NF- $\mathrm{KB}$ activator (TANK)-binding kinase 1 (TBK1) and the inducible IKB kinase (IKK-i), ultimately leading to activation of transcription factors, such as NF- $\mathrm{KB}$, interferon-regulatory factor (IRF), to induce immune or inflammatory responses. In the past few years, immunologists have demonstrated the central role of TRAFs in inflammation, innate immunity, and regulation of NF- $\mathrm{kB}$ pathway, as well as type 1 interferon expression. However, many aspects on TRAFs func-

email: zhengdx@pumc.edu.cn tion and molecular mechanism remain to be further clarified.

Prof. Liu Yang et al., who provided the first experimental evidence that pathogen recognition by the innate immune system turns on the adaptive immune system [3], summarized how the immune system controls the balance between recognizing pathogen-associated molecular patterns (PAMPs) and danger-associated molecular patterns (DAMPs) [4]. They discovered a novel pathway mediated by CD24 and its receptor, Siglec G, in mouse or Siglec 10 in human, which deepens our understanding on how host establishes sterilizing immunity to most pathogens and avoids fatal damage in response to aseptic tissue injury. The CD24-mediated pathway suppresses host response to DAMPs, but not to PAMPs, therefore, allowing the host to distinguish PAMPs from DAMPs. Blocking this pathway results in a devastating systemic inflammatory response when necrosis occurs in mouse model. Therefore, CD24/Siglec 10 pathway enables a cross-reactive pattern recognition to initiate immunity against pathogens without significant immune-mediated self-destruction in case of tissue injury. However, the cross-talk between CD24 receptor-mediated pathway and TLR signaling is remained to be further studied.

Tang Fei in Prof. Liu YongJun's group, who discovered plasmacytoid dendritic cells (pDCs) about 10 years ago $[5,6]$, presented a review on the role of pDCs in anti-viral immunity and autoimmunity [7]. The pDCs are capable to express large amount of type I interferons (IFNs) in response to viral infection, since they express TLR7 and TLR9, which recognize viral nucleic acids in the endosomal compartment. The pDCs-expressed type I IFNs not only 
suppress viral replication directly but also play an important role in the regulation of innate and adaptive immunity. Aberrant activation of pDCs by self nucleic acids and the subsequent production of type I IFNs are also observed in some autoimmune diseases. So that, pDC could be an attractive therapeutic target for the vial infection and autoimmune diseases. Whereas, Tang et al. pointed out in this review article that specific and detailed pathway for pDC development, and how to develop a safe and effective immuno-therapeutics for viral infectious and autoimmune diseases need to be further investigated.

Wang ShengDian in Prof. Chen LiePing's group, who discovered various co-stimulatory molecules in $\mathrm{T}$ cell activation [8], contributed a review on the structural immunology of co-stimulatory and co-inhibitory molecules [9]. Co-stimulatory signaling pathways in T cells play a central role in regulating immune responses. Targeting these pathways represents one of the most promising approaches for the development of immuno-therapeutics. The structure of costimulatory molecule reveals invaluable mechanistic insights underlying costimulatory receptor/ligand specificity, affinity, oligomeric state, and valency, and provides an extraordinary opportunity for the development of immunotherapy for T cell co-stimulatory pathway-related disorders.

Prof. Tang Hong and $\mathrm{Fu}$ YangXin introduced their recent finding [10] on $\mathrm{T}$ cells in dampening innate inflammatory responses [11]. This finding suggests that $\mathrm{T}$ cells of adaptive immunity regulate innate responses, therefore, shed new light on the evolution and interaction between innate and adaptive immunity. Further understanding of the underlying mechanisms will help us to make better clinical diagnosis and treatment for various infectious and inflammatory diseases.

Finally, Zhang LiGuo in Prof. Chen JianZhu and $\mathrm{Su}$ LiShan's group contributed the last article on establishment of a new generation of humanized mouse for studying human immunology and HIV-1 immuno-pathogenesis in vivo [12]. They described the criteria of robust animal model for hypothesis testing and mechanistic research in human immunology and immunopathology should be met, and sum- marized the current understanding of HIV-1 immunopathogenesis in patients and in SIV-infected primate models as well as the recent progress in the development of humanized mouse model, especially the immunodeficient mouse that carries a defective gammaC gene. These mouse models lack NK, T and B cells, which are crucial for the in vivo study of human immunology and stem cell biology in tissue development and functional expression.

Immunology has become an integral component of medical science, deepening our understanding for the pathophysiology of human diseases, and has contributed greatly to the development of therapeutics for infection, autoimmune diseases, allergy and cancer. I believe that these reviews above are valuable for both Chinese communities of immunology and medicine.

1 Cheng G, Cleary A M, Ye Z S, et al. Involvement of CRAF1, a relative of TRAF, in CD40 signaling. Science, 1995, 267: 1494-1498

2 Wang Y Y, Zhang P, Liu Y F, et al. TRAF-mediated regulation of immune and inflammatory responses. Sci China Life Sci, 2010, 53: 159-168

3 Chen G Y, Tang J, Zheng P, et al. CD24 and siglec-10 selectively repress tissue damage-induced immune responses. Science, 2009, 323: 1722-1725

4 Liu Y, Chen G Y, Zheng P, et al. On self-nonself discrimination in pattern recognition. Sci China Life Sci, 2010, 53: 169-171

5 Siegal F P, Kadowaki N, Shodell M, et al. The nature of the principal type 1 interferon-producing cells in human blood. Science, 1999, 284: 1835-1837

6 Liu Y J. IPC: Professional type 1 interferon-producing cells and plasmacytoid dendritic cell precursors. Annual Rev Immunol, 2005, 23: $275-306$

7 Tang F, Du Q M, Liu Y J. Plasmacytoid dendritic cells in antiviral immunity and autoimmunity. Sci China Life Sci, 2010, 53: 172-182

8 Chen L P. Co-inhibitory molecules of the B7-CD28 family in the control of T-cell immunity. Nat Rev Immunol, 2004, 4: 336-347

9 Wang S D, Chen L P. Structural immunology of costimulatory and coinhibitory molecules. Sci China Life Sci, 2010; 53: 183-189

10 Kim K D, Zhao J, Yang X, et al. Adaptive immune cells temper initial innate responses. Nat Med, 2007, 13: 1248-1252

11 Tang H, Fu Y X. A new role for T cells in dampening innate inflammatory responses. Sci China Life Sci, 2010, 53: 190-194

12 Zhang L G, Meissner E, Chen J Z, et al. Current humanized mouse models for studying human immunology and HIV-1 immunopathogenesis. Sci China Life Sci, 2010, 53: 195-203

Open Access This article is distributed under the terms of the Creative Commons Attribution License which permits any use, distribution, and reproduction in any medium, provided the original author(s) and source are credited. 